Universidad de La Salle

Ciencia Unisalle

Utopía - Working papers

Utopía

2020-12-01

\title{
Evaluación de métodos de control para el chinche patifoliado (Leptoglossus zonatus) en badea (Passiflora quadrangularis)
}

\author{
Javier Salazar \\ Universidad de La Salle, Bogotá \\ Dixon Carrascal \\ Universidad de La Salle, Bogotá \\ Duvan Santamaria \\ Universidad de La Salle, Bogotá \\ Karen Cárdenas \\ Universidad de La Salle, Bogotá \\ Liced Paz Alegría \\ Universidad de La Salle, Bogotá
}

See next page for additional authors

Follow this and additional works at: https://ciencia.lasalle.edu.co/utopia_working_papers

\section{Recommended Citation}

Salazar, Javier; Carrascal, Dixon; Santamaria, Duvan; Cárdenas, Karen; Paz Alegría, Liced; Peña, Katherin; and Chavarro, Yen Franklin, "Evaluación de métodos de control para el chinche patifoliado (Leptoglossus zonatus) en badea (Passiflora quadrangularis)" (2020). Utopía - Working papers. 8.

https://ciencia.lasalle.edu.co/utopia_working_papers/8

This Documento de trabajo is brought to you for free and open access by the Utopía at Ciencia Unisalle. It has been accepted for inclusion in Utopía - Working papers by an authorized administrator of Ciencia Unisalle. For more information, please contact ciencia@lasalle.edu.co. 


\section{Autor}

Javier Salazar, Dixon Carrascal, Duvan Santamaria, Karen Cárdenas, Liced Paz Alegría, Katherin Peña, and Yen Franklin Chavarro

This documento de trabajo is available at Ciencia Unisalle: https://ciencia.lasalle.edu.co/utopia_working_papers/8 
https://doi.org/10.19052/wp.utopia.2020.4

\title{
Utopía | 10 \\ Working Papers \\ 2020
}

\section{Evaluación de métodos de control para el chinche patifoliado (Leptoglossus zonatus) en badea (Passiflora quadrangularis)}

\author{
Javier Salazar ${ }^{1}$, Dixon Carrascal ${ }^{2}$, Duvan Santamaria ${ }^{2}$, Karen Cárdenas ${ }^{2}$, Liced Paz Alegría ${ }^{2}$, Katherin Peña ${ }^{2}$, Yen Frankinl \\ Chavarro $^{2}$
}

\section{RESUMEN}

La badea (Passiflora quadrangularis) es una especie perteneciente a la familia pasiflorácea, la cual se adapta a zonas tropicales y subtropicales. Este cultivo viene trabajándose en la línea de Fruticultura del Campus Utopía como cultivo promisorio. Sin embargo, ha presentado un problema fitosanitario, el ataque de un chinche de la familia Coreidae. Esta plaga perfora la epidermis y succiona la savia del fruto generando puntos necróticos, los cuales sirven como puerta de entrada de hongos saprofitos, lo cual genera el deterioro de la calidad del fruto y afecta su crecimiento. En esta investigación se llevó a cabo la identificación taxonómica de la plaga, se determinó su ciclo de vida y se evaluaron diferentes métodos de control para su control (dos insecticidas de síntesis química y el hongo entomopatógeno, Beauveria bassiana), a partir del establecimiento de una cría con adultos capturados en campo. La identificación del insecto indico que se trata de la especie Leptoglossus zonatus. Las hembras depositaron 20 huevos en promedio y tras 9 días, hubo una emergencia de ninfas del 100\%. Los insecticidas Clorpirifos e Imidacloprid mostraron un 100\% de mortalidad en comparación al $40 \%$ del hongo entomopatógeno. Beauveria bassiana evaluado en diferentes dosis, registro que en dosis de $4 \mathrm{~g} / \mathrm{l}$ alcanza un $50 \%$ de mortalidad. A pesar del éxito del control químico, no se puede descartar la acción de B. bassiana y su inclusión en programas de manejo integrado de L. zonatus en Badea.

Palabras claves: Passifloraceae, Coreidae, Plaga, Ovoposición, Control biológico.

Evaluation of control methods for the leaf-footed bug (Leptoglossus zonatus) in giant granadilla (Passiflora quadrangularis)

\begin{abstract}
The giant granadilla (Passiflora quadrangularis) is a species belonging to the Passifloraceae family, which is adapted to tropical and subtropical areas. This crop has been working in the Fruit Growing line of the Utopia Campus as a promissory crop. However, it has presented a phytosanitary problem, the attack of a bug of coreidae family. This pest pierces the epidermis and sucks the sap from the fruit, generating necrotic points, which serve as entry points for saprophytic fungi, which deteriorates the quality of the fruit and affects its growth. In this research, the taxonomic identification of the pest was carried out, its life cycle was determined and different control methods were evaluated for
\end{abstract}

${ }^{1}$ Docente del programa de ingeniería Agronómica de la Universidad de La Salle

${ }^{2}$ Estudiante de ingeniería Agronómica de la Universidad de la Salle 


\section{Utopía | 10 \\ Working Papers \\ 2020}

its control (two chemical synthesis insecticides and the entomopathogenic fungus, Beauveria bassiana), from the establishment of a rearing with collected in field. The identification of the insect indicated that it is the Leptoglossus zonatus species. The females laid an average of 20 eggs and after 9 days, there was a $100 \%$ nymphs emergence. Chlorpyrifos and Imidacloprid insecticides showed $100 \%$ mortality compared to $40 \%$ of the entomopathogenic fungus. Beauveria bassiana evaluated in different doses, showed that the doses of $4 \mathrm{~g} / 1$ reached of $50 \%$ mortality. Despite the success of the chemical control, the action of B. bassiana and its inclusion in integrated management programs of $\mathrm{L}$. zonatus in giant granadilla cannot be ruled out.

Keywords: Passifloraceae, Coreidae, Pest, Oviposition, Biological control

\section{INTRODUCCIÓN}

La badea (Passiflora quadrangularis) es una especie trepadora perteneciente a la familia de las Passifloraceae, la cual se adapta a zonas tropicales y subtropicales; la zona de origen aun no es muy clara, pero expertos indican que es procedente de América del Sur, de países como Perú, Brasil y las islas del caribe en donde se encuentran especies afines (Duque y Morales, 2005).

En Colombia el cultivo de badea se encuentra priorizado por el Ministerio de Agricultura y Desarrollo Rural (MADR) como cultivo promisorio para exportación por su aceptación para consumo en fresco, jugos o aperitivos (Carranza, Castellanos, Deaza, y Miranda, 2016). Según el MADR (2015), en el 2013 había en Colombia de 431 ha sembradas con una producción de 3.881 t y rendimiento de 14,8 t/ha. Asimismo, este cultivo brinda un aporte económico, ya que es una fruta que constituye una alternativa viable para agricultores de pequeña y mediana escala, como también para empresas con visión industrial (Zamora, 2015).

En el cultivo de badea ( $P$. quadrangularis) se presentan plagas y enfermedades que limitan el óptimo crecimiento de los frutos. De esta manera, en la línea de fruticultura del Campus Utopía, se ha está presentando un problema causado por un chinche perteneciente a la familia coreidae. Este insecto perfora la epidermis y succiona la savia del fruto, ataque realizado tanto por los adultos como por los inmaduros (ninfas). Su ataque genera puntos necróticos, los cuales sirven como puntos de entrada de hongos saprofitos como Collethotricum sp. (Reina,1996), lo cual genera el deterioro de la calidad del fruto y afecta su crecimiento, y pueden reducir el número de semillas en el fruto (Barrera, Calyecac, Castrejón y Sánchez, 2016). 


\section{Utopía | \\ Working Papers \\ 2020}

Uno de los géneros más importante dentro de la familia coreidae es Leptoglossus (Guérin-Méneville), el cual se considera polífago y con registro de ataque en cultivos como maíz (Zea mays), algodón (Gossypium hirsutum), melocotón (Prunus pérsica), cítricos (Citrus), sandia (Citrullus lanataus) y sorgo (Sorghum) (Allen, 1969; Solomon y Froeschner, 1981; Schaefer y Mitchell, 1983; Tepole, Rojas, Reyes y Castrejón, 2016). Es un insecto hemimetábolo y dependiendo de las condiciones ambientales y alimentación su ciclo de vida varia. El insecto pasa por 5 estadios ninfales antes de llegar al estado adulto (Tepole, 2011).

De esta manera, la presenta investigación tuvo por objetivos realizar la debida identificación de las especies, conocer aspectos básicos de su ciclo de vida y evaluar diferentes métodos de control, que permitan disminuir las pérdidas generadas por este.

\section{METODOLOGÍA}

El ensayo se llevó a cabo en el laboratorio de entomología de la Universidad De La Salle campus Utopía (5 $19^{\prime}$ $31^{\prime \prime}$ latitud Norte y $72^{\circ} 17^{\prime} 48^{\prime \prime}$ longitud Oeste). Para el establecimiento de la cría en laboratorio, se realizó la recolección de adultos del chinche en el cultivo de badea. Los adultos se dispusieron en cajas de madera (60 x 60 x $60 \mathrm{~cm}$ ); con paredes cubiertas con tela tipo tul. En una primera se depositó una pareja de chinches adultos y 6 ninfas, en una segunda caja se introdujeron dos parejas, las cuales se capturaron copulando y en una tercera caja se dispusieron 8 chinches adultos; cabe anotar que los chinches de la caja 1 y 3 no fueron sexados. Inicialmente, se ofrecieron como alimento: maíz choclo (Zea mays), mandarina (Citrus reticulata) y agua miel, pero por cuestiones económicas, la alimentación ofrecida finalmente fue frutos de badea y agua miel. Monitoreos diarios fueron realizados para obtener información sobre aspectos del ciclo biológico de la especie. Se registraron los datos de temperatura mínima y máxima, pues esta variable ambiental juega un papel clave en la longitud del ciclo de vida de todo insecto. La identificación taxonómica fue realizada por el profesor Fredy Alexander Rodríguez, con base en la clave dicotómica de Brailovsky (2014). 


\section{Utopía | \\ Working Papers \\ 2020}

En condiciones de laboratorio se evaluaron dos insecticidas y un hongo entomopatógeno. Se usaron cinco tratamientos, en el primer tratamiento se utilizó Lorsban ${ }^{\circledR} 4$ EC (ingrediente activo Clorpirifos) a una dosis de 1ml/1; en el tratamiento dos se utilizó Agridor 350 S.C. (ingrediente activo Imidacloprid) con una dosis de 1m/l; en el tercer tratamiento se utilizó Agroin -B (Beauveria bassiana) a una dosis de 2g/l; en el tratamiento cuatro se utilizó agua y en el quinto tratamiento no se realizó ningún tipo de aplicación.

Cinco adultos sin sexar fueron usados por cada tratamiento, aislados en cajas diferentes a las de cría. La aplicación se realizó por medio de atomizadores, asegurándose de que los adultos fueran cubiertos por cada producto según el tratamiento. A partir de la aplicación, se realizó un monitoreo durante 6 días, registrando el número de insectos muertos por día y se realizó una curva de mortalidad.

Como un tercer paso y debido al resultado obtenido en el tratamiento el hongo entomopatógeno. Se realizo la recolección de chinches adultos en campo, los cuales se aislaron en cajas (30 cm x $30 \mathrm{~cm}$ x $20 \mathrm{~cm}$ ), siendo mantenidos por tres días bajo observación, para evidenciar muerte natural. Fueron evaluados cinco tratamientos: en el primer tratamiento se utilizó agua, en el segundo tratamiento se utilizó como producto comercial Agroin- B (Beauveria bassiana) en una dosis de $1 \mathrm{~g} / \mathrm{l}$; en el tercer tratamiento no se realizó ningún tipo de aplicación; en el cuarto tratamiento se utilizó Agroin- B en dosis de 2 g/l y en el quinto tratamiento Agroin-B en dosis de $4 \mathrm{~g} / \mathrm{l}$.

Fueron usados 10 adultos sin sexar por cada tratamiento. El diseño experimental fue completamente al azar. Para la aplicación de los productos se utilizó la misma metodología mencionada anteriormente. El registro de la mortalidad se llevó a cabo durante 18 días y finalmente se realizó la curva de mortalidad.

\section{RESULTADOS Y DISCUSIÓN}

La identificación del chinche coreidae que ha atacado la badea resulto en la determinación de la especie Leptoglossus zonatus (Dallas, 1852) (figura 1). 


\section{Utopía | \\ Working Papers \\ 2020}

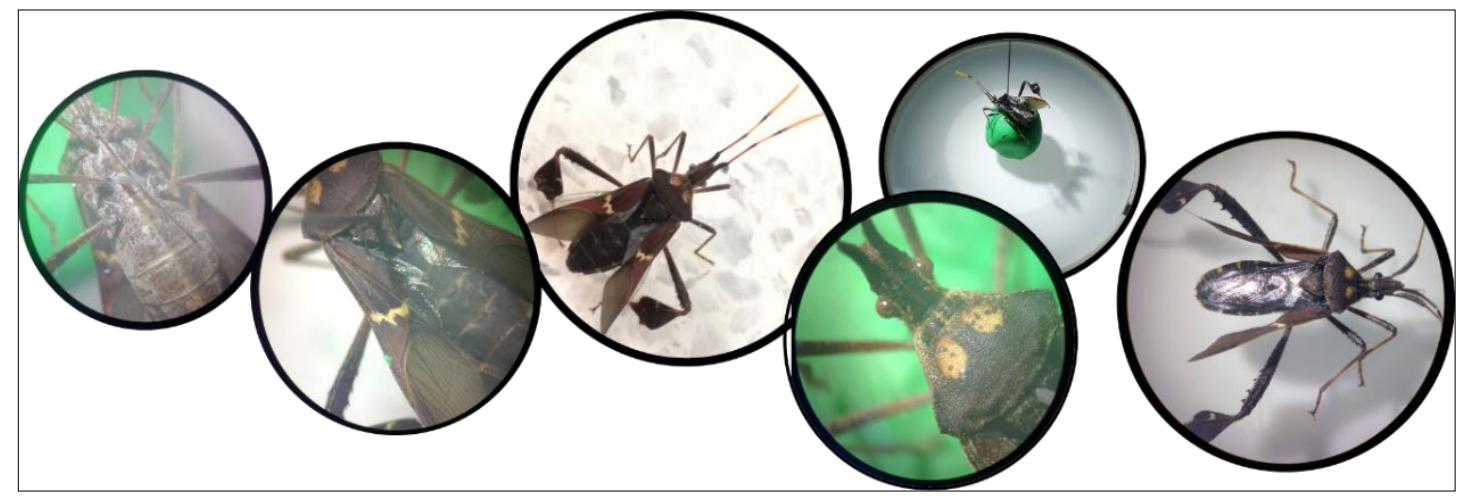

Figura 1. Características morfológicas de la especie Leptoglossus zonatus. Puntos amarillos en el pronoto, líneas aserradas amarillas en los hemélitros y antenas bicolor.

La alimentación de L. Zonatus con badea y agua miel en condiciones de laboratorio con una temperatura mínima de $28^{\circ} \mathrm{C}$ y máxima de $31^{\circ} \mathrm{C}$, resulto en los siguientes datos de ciclo de vida: los huevos tardaron 9 días en eclosionar, hay cinco estados ninfales los cuales variaron en duración individual de 5 a 10 días aproximadamente. Las ninfas de segundo y quinto estadio registraron la mayor duración.

El tiempo total del estadio ninfal fue de 43 días. La longevidad del adulto fue de 83 días. El ciclo completo de vida fue de 126 días (figura 2). Registros del ciclo de vida de la especie en México obtuvieron un ciclo de vida de 109 días (Tepole, 2011, Tepole et al., 2016). Sin embargo, los autores mencionan que los periodos de desarrollo de cada uno de los estadios ninfales dependen de factores como la temperatura y el tipo de alimentación. 


\section{Utopía | 10}

Working Papers

2020

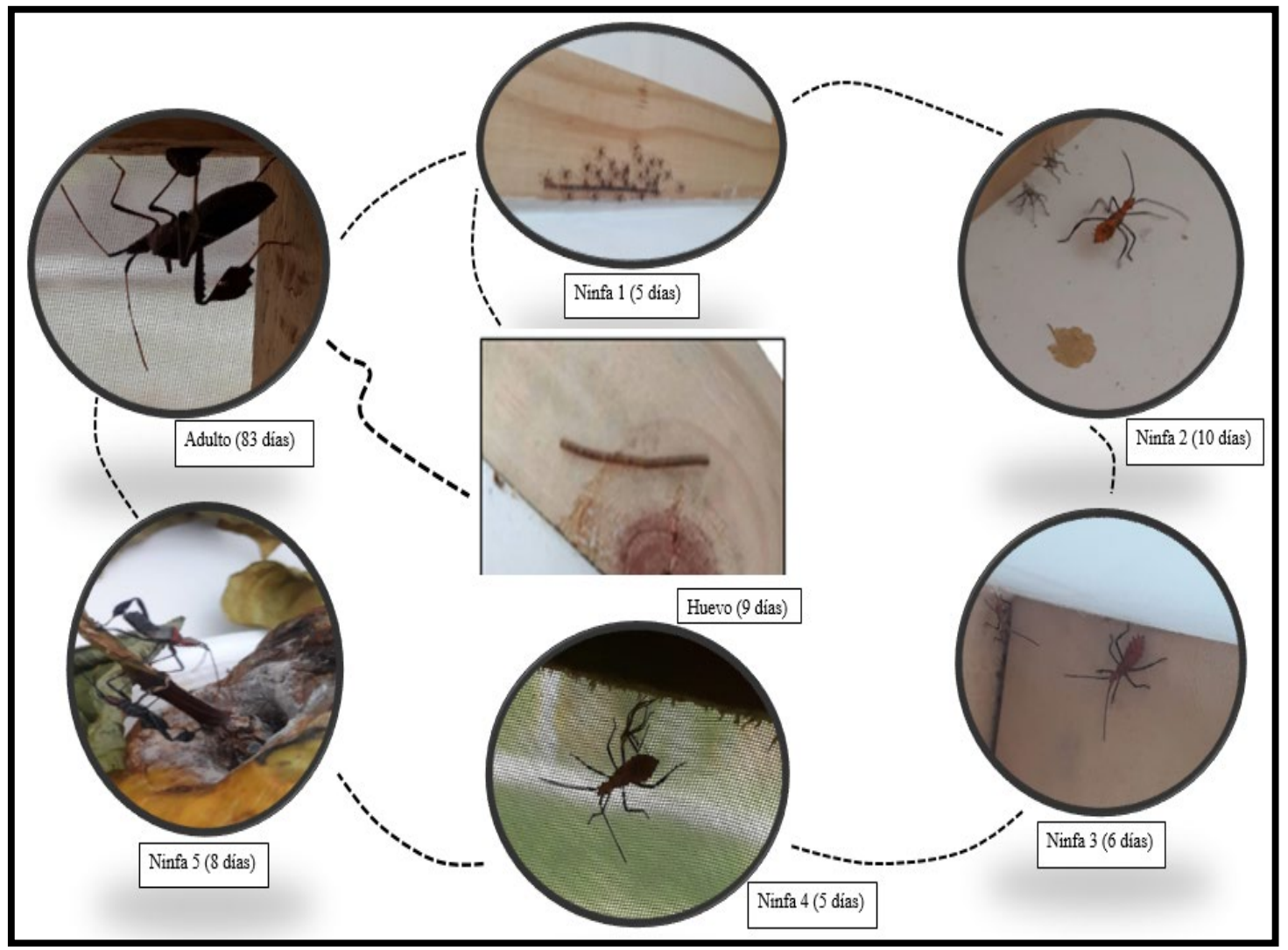

Figura 2. Ciclo de vida de Leptoglossus zonatus alimentado en badea y agua miel.

En la evaluación inicial de métodos de control, se pudo evidenciar que el tratamiento con Beauveria bassiana, produjo una mortalidad del $40 \%$ hasta el día 6. Beauveria bassiana ha sido utilizada específicamente como un agente de control biológico de L. zonatus, con un alto porcentaje de mortalidad, del 90\%, actuando entre los 7 - 10 días después de su aplicación (Barrera et al., 2016).

Para el control de L. zonatus, se utilizan tradicionalmente insecticidas químicos y hongos entomopatógenos. Sin embargo, el uso de este último no se reporta a gran escala, pues los insecticidas de síntesis química actúan en un menor tiempo que los biológicos (Tepole et al., 2016). Los tratamientos 1 y 2 causaron una mortalidad del 100\% en 1- 2 días, mostrándose más efectivos que el control con hongos entomopatógenos (figura 3). 


\section{Utopía | \\ Working Papers \\ 2020}

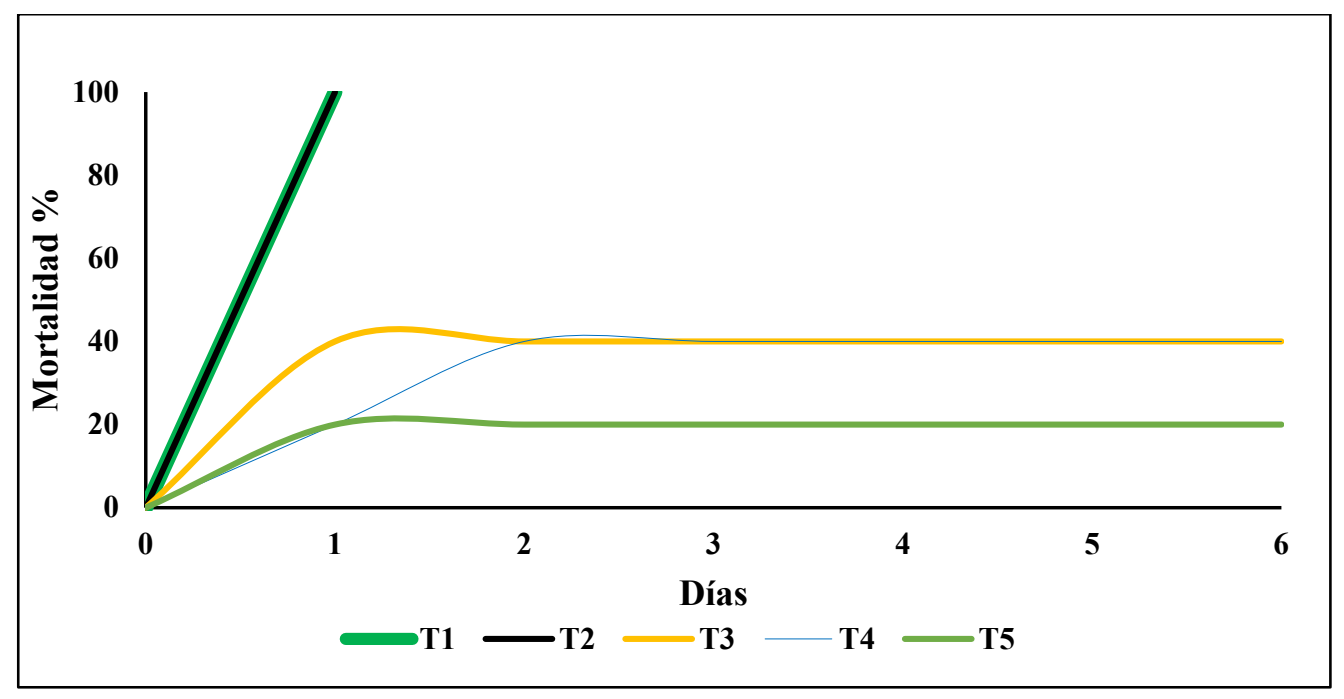

Figura 3. Mortalidad de los diferentes métodos de control evaluados en Leptoglossus zonatus

De acuerdo con Malpartida-Zevallos (2013), B. bassiana es producido en muchos países y se usa principalmente en el control de lepidópteros y coleópteros. Los síntomas de su ataque en insectos son la pérdida de sensibilidad, falta de coordinación, letargo, inapetencia, melanización y parálisis. Al morir el insecto atacado, se presenta la esporulación y la posterior dispersión del hongo, permitiendo un control más allá de la aplicación.

Al evaluar diferentes dosis de B. bassiana, se evidencio que en el día 7 el hongo empezó a presentar los efectos de su ataque, debido a que en este día en el tratamiento 5 (4 g/l) se registró la muerte de dos individuos. Según Barrera (2016), B. bassiana puede ejercer un control efectivo en L. zonatus debido a su alta tasa de mortalidad obtenida en el ensayo previo en los días 7 y 8 , aunque también difieren en que la importancia de un control efectivo no es la alta tasa de mortalidad, sino que se logre una alta mortalidad con cierta rapidez.

También se puede evidenciar que B. bassiana en el tratamiento de menor dosis que corresponde al tratamiento 4 (1g/l), empezó a tener efecto en el día 9. Según Góngora (2013), B. bassiana en condiciones ideales para la colonización de humedad, temperatura y luz, en aproximadamente ocho días ha completado las tres etapas del ciclo de colonización y hace efecto en la plaga. Entretanto, Bustamante (2019) afirma que los mayores índices de mortalidad causada por B. bassiana se presentan entre los días 7 a 9 después de su aplicación. 


\section{Utopía | \\ Working Papers \\ 2020}

Godoy et al. (2007), encontraron que B. bassiana causa una mayor mortalidad a $25^{\circ} \mathrm{C}$. En este ensayo, la temperatura mínima tuvo un promedio de $28,5^{\circ} \mathrm{C}$. Los mismos autores afirman que B. bassiana se desarrolla en óptimas condiciones entre $25^{\circ} \mathrm{C}$ y $30^{\circ} \mathrm{C}$.

Los tratamientos evaluados con diferentes dosis de B. bassiana presentaron la siguiente mortalidad: T1 (agua), 20\% de mortalidad; T4: B. bassiana (dosis de 1 g/l) 10\% de mortalidad; T5: B. bassiana (dosis de 4 g/l), 50\% de mortalidad; T2: B. bassiana (dosis 2 g/l) 40\% de mortalidad. Barrera et al. (2016), reportan que la mortalidad de adultos de L. zonatus con tres diferentes cepas de B. bassiana alcanzó un $50 \%$ entre 10 y 13 días después de la aplicación.

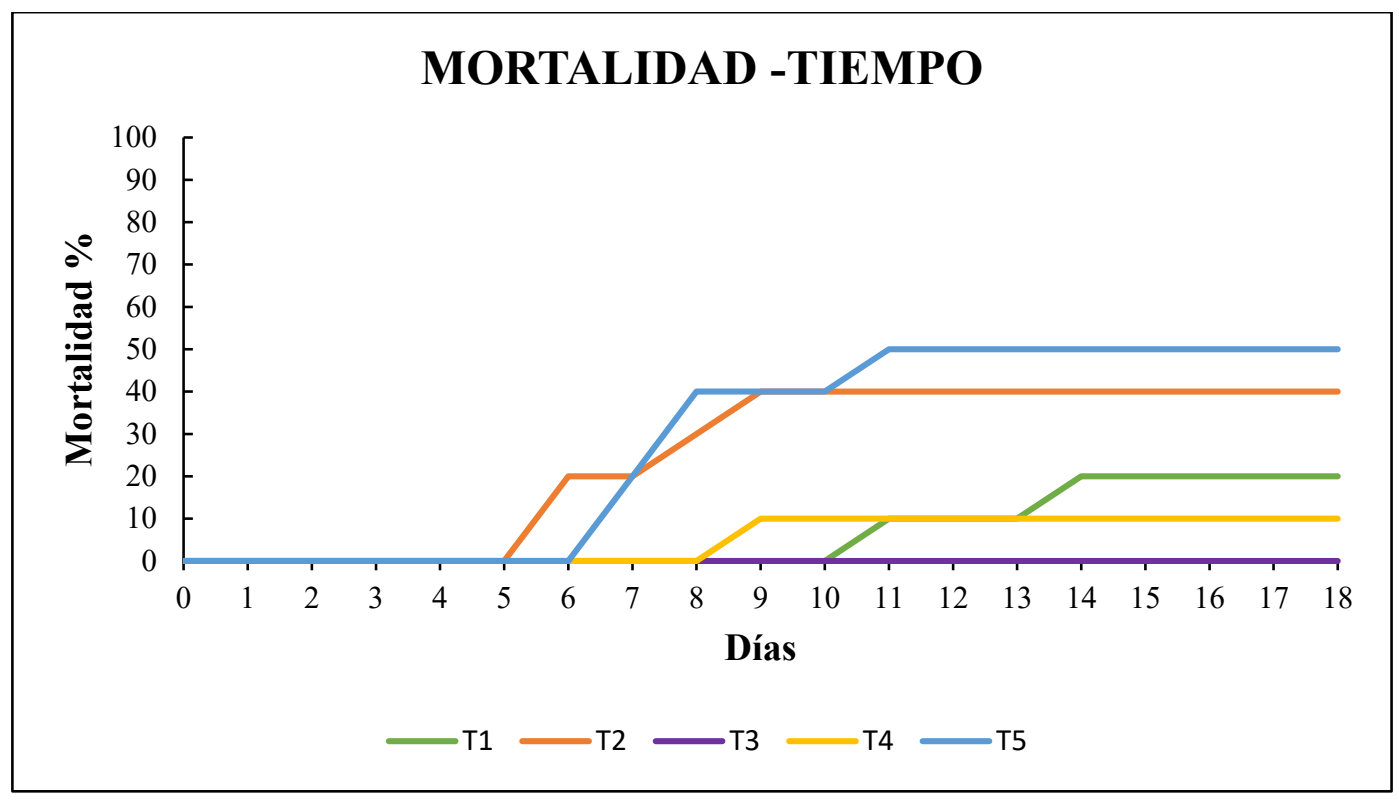

Figura 4. Mortalidad en diferentes dosis de Beauveria bassiana.

\section{CONCLUSIONES}

Se determinó que la especie de chinche coreidae que ha venido atacando el cultivo de badea en el Campus Utopía pertenece a Leptoglossus zonatus. El cual en las condiciones de Yopal presenta un ciclo de vida de 126 días. 
https://doi.org/10.19052/wp.utopia.2020.4

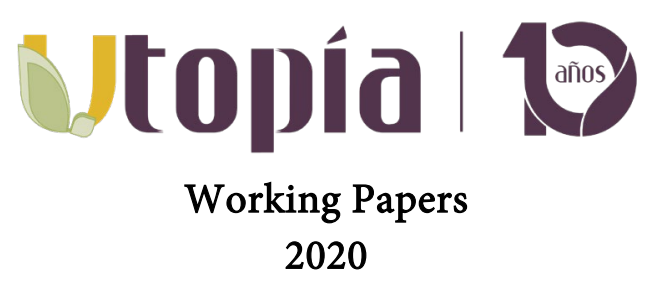

Los métodos evaluados para su control evidenciaron que puede ser manejado con los ingredientes activos Clorpirifos e Imidacloprid, los cuales presentaron un 100\% de mortalidad en comparación al 40\% del hongo entomopatógeno Beauveria bassiana. Sin embargo, B. bassiana en dosis de 4g/l alcanza una mortalidad del 50\%.

De esta manera, a pesar del éxito del control químico, no se puede descartar la acción de $B$. bassiana y su inclusión en programas de manejo integrado de L. zonatus en Badea.

\section{BIBLIOGRAFÍA}

Allen, R. (1969). Una revisión del género Leptoglossus Guerin (Hemiptera: Coreidae). Entomol. A.m. 45: 35 140.

Barrera, O., Calyecac, G., Castrejon, V., Sánchez, J. (2016). Control biológico de la chinche pata de hoja, plaga del piñón de cerro en condiciones de laboratorio.

Barta, M. (2016). Biology and temperature requirements of the invasive seed bug Leptoglossus occidentalis (Heteroptera: Coreidae) in Europe. Journal of pest science, 89(1), 31-44.

Brailovsky, H. (2014). Illustrated key for identification of the species included in the genus Leptoglossus (Hemiptera: Heteroptera: Coreidae: Coreinae: Anisoscelini), and descriptions of five new species and new synonyms. Zootaxa, 3794(1), 143-178.

Carranza, C., Castellanos, G., Deaza, D., Miranda, D. (2016). Efecto de la aplicación de reguladores de crecimiento sobre la germinación de semillas de badea (Passiflora quadrangularis L.) en condiciones de invernadero. Revista Colombiana de Ciencias Hortícolas, 10(2), 284-291.

Cuyo, M. (2017). Producción de badea (Passiflora quadragularis) con la aplicación de abonos orgánicos líquidos (Bachelor's thesis, La Maná: Universidad Técnica de Cotopaxi; Facultad de Ciencias Agropecuarias y Recursos Naturales; Carrera de Ingeniería Agronómica).

Duque, C., Morales, A. (2005). El aroma frutal de Colombia. Ilustrada ed. Colombia. pp. 107-130. 


\section{Utopía | \\ Working Papers \\ 2020}

Galarza, E. (2016). Incidencia de la mosca del botón floral (Dasiops inedulis) y el chinche patón (Leptoglosus zonatus) en el cultivo de maracuyá (Passiflora edulis var. Flavicarpa) en la zona de Quevedo. Universidad Técnica Estatal de Quevedo Facultad de Ciencias Agrarias Carrera de Ingeniería Agronómica.

Godoy, J. C., Valera, R. E., Guédez, C., Cañizalez, L. M., Castillo, C. (2007). Determinación de temperatura y humedad óptima para la germinación y esporulación de cinco aislamientos de Beauveria bassiana. Revista Facultad de Agronomía (LUZ), 24(3), 415-425.

González-Santarosa, M. G., Salazar-Torres, J. C., Jaimes-Albíter, F., Ramírez-Alarcón, S., González-Santarosa, R. (2010). Eficacia de Beauveria bassiana (Balsamo) Vuillemin en el control de Lygus lineolaris (Palisot de Beauvois) en fresa. Revista Chapingo. Serie horticultura, 16(3), 189-193

Góngora, C. E., Marín, P., Benavides, P. (2013). Claves para el éxito del hongo Beauveria bassiana como controlador biológico de la broca del café. Centro Nacional de Investigaciones de Café (Cenicafé).

Malpartida-Zevallos, J., Narrea-Cango, M., Dale-Larraburre, W. (2013). Patogenicidad de Beauveria bassiana (Bals) Vuill., sobre el gusano defoliador del maracuyá Dione juno (Cramer) (Lepidoptera: Nymphalidae) en laboratorio. Ecología Aplicada, 12(2), 75-81.

MADR. (2015). Boletín cadena de pasifloras. En: Sistema de Información de Gestión y Desempeño de Organizaciones de Cadenas. Ministerio de Agricultura y Desarrollo Rural, Bogotá.

Panizzi, A (1989). Desempeño de ninfas y adultos de Leptoglossus zonatus (Dallas, 1852) (Hemiptera: Coreidae) en diferentes alimentos. Un. Soc. Entomol. Bras. 18: 375-389.

Reina, E. (1996). Manejo postcosecha y evaluación de la calidad para la badea (Passiflora cuadrangulares) que se comercializa en Neiva.

Schaefer, C., Mitchell, P. (1983). Plantas alimenticias de Coreoidea (Hemiptera: Heteroptera). Ana. Entomol. Soc. A.m. 76: 591-615. 


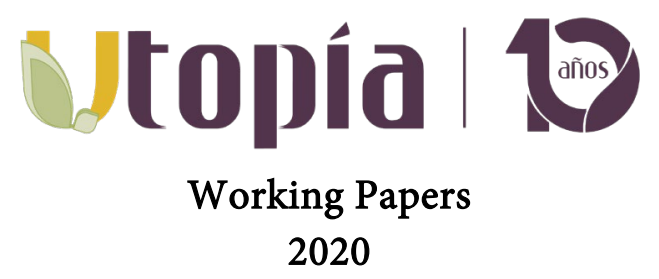

Solomon, J., Froeschner, R. (1981). Notas sobre los recursos alimentarios y el comportamiento de la familia Coreidae (Hemiptera) en un bosque tropical semi-caducifolio. Proc. Entomol. Soc. Wash. 83: 428-431.

Tepole, R. (2011). Ciclo biológico y análisis de riesgo de Leptoglossus zonatus DALLAS (HETEROPTERA: COREIDAE) para el sorgo en el estado de Morelos (Doctoral dissertation).

Tepole, R., Rojas, S., Reyes, J., y Castrejón, V. (2016). Ciclo de vida y análisis de riesgo climático de Leptoglossus zonatus Dallas (Hemiptera: Coreidae) para las zonas productoras de sorgo en el estado de Morelos, México. Acta zoológica mexicana, 32(3), 300-309.

Toapanta, A. (2018). Caracterización morfológica y evaluación preliminar de tomate de árbol (Solanum betaceum Cav) a Leptoglossus zonatus (Hemiptera: Coreidae) (Bachelor's thesis, Quito: UCE).

Zamora, A. (2015). Determinación de las propiedades físicas y químicas de la badea (Passiflora quadrangularis). Trabajo de Investigación, Universidad Técnica de Ambato, Facultada de Ciencias e Ingeniería en Alimentos, Ambato. 